\title{
Remoción de nutrientes mediante coagulantes naturales y químicos en planta de tratamiento de aguas residuales, Valledupar Colombia
}

\section{Nutrient Removal using natural and chemical coagulants in waste water treatment plant, Valledupar Colombia}

\section{Remoção de nutrientes por coagulantes naturais e químicas em águas residuais estação de tratamento, Valledupar Colômbia}

\author{
Damileth Dearmas Duarte ${ }^{1}$ \& Luis Francisco Ramírez Hernández² \\ ${ }^{1}$ Ingeniera Ambiental y Sanitaria, Magister en Ciencias ambientales, Estudiante de Doctorado en \\ Estudios Urbanos de la Universidad Nacional de General Sarmiento. Buenos Aires. Argentina. \\ ${ }^{2}$ Ingeniero Civil, Especialista y Magister en Ingeniería Sanitaria y Ambiental.
}

${ }^{1}$ Programa de Doctorado en Estudios Urbanos de la Universidad Nacional de General Sarmiento. Buenos Aires. Argentina. ${ }^{2}$ Departamento Ingeniería Ambiental y Sanitaria.

Universidad del Cesar. Valledupar. Colombia

1ingeambientedamileth@hotmail.com, ${ }^{2}$ luisfranciscoramirez@unicesar.edu.co

\section{Resumen}

Se evaluó desempeño y eficiencia de dos coagulantes químicos: sulfato de aluminio y policloruro de aluminio (PAC), y dos naturales: moringa (Moringa oleífera) y cardón guajiro (Lemaireoreus griseus) en la remoción de nutrientes y sedimentos del efluente de la planta de tratamiento de aguas residuales conformado por lagunas de estabilización El Salguero ubicada en el municipio de Valledupar, Cesar, Colombia. Los ensayos se llevaron a cabo con muestras de agua procedentes del río Cesar recogidas en el efluente del sistema de tratamiento. La turbidez varía desde 25 NTU hasta 67 NTU durante la época de desarrollo de la investigación. Se obtuvo que el sulfato de aluminio pese a no alcanzar la turbidez exigida por la normatividad colombiana (Decreto 3930/2010, Articulo N72 Turbidez $\geq 5$ NTU) es un coagulante que remueve más del $80 \%$ de los parámetros de DQO,
DBO y algas. El policloruro de aluminio es adecuado para disminuir significativamente la turbidez y los SST en un 92\%, además presentó la mejor viabilidad económica de los tratamientos; y para la disminución de fosforo total resultaron efectivos los tratamientos con coagulantes naturales moringa y cardón, los cuales presentaron una remoción de $90.35 \%$. Se concluye que los coagulantes evaluados presentan un potencial de remoción de los parámetros analizados, los resultados avalan a la Moringa oleífera como una posible alternativa natural en la potabilización de las aguas, ya que garantiza no sólo lograr con éxito el proceso de coagulación, sino también, la ausencia de residuos.

Palabras clave: agua residual, efluente, nutrientes, eutrofización, prueba de jarras. 


\section{Abstract}

Performance and efficiency of two chemical coagulants aluminum sulfate and aluminum polychloride (PAC), and two natural: moringa (Moringa oleifera) and cardon guajiro (Lemaireoreus griseus) in the removal of nutrients and sediments of the effluent from the waste water treatment plant were assessed; the effluent consisted of stabilization ponds in the Salguero located in the municipality of Valledupar, $\mathrm{Ce}$ sar, Colombia. The tests were carried out with water samples from the Cesar River collected in the effluent of the treatment system. The turbidity varied from 25 NTU to 67 NTU during the time of development of the research. The results showed the aluminum sulfate despite not achieving the turbidity required by Colombian Law (Decree 3930/2010, Article №72 Turbidity $\geq$ $5 \mathrm{NTU}$ ) is a coagulant that removed more than $80 \%$ of $\mathrm{COD}, \mathrm{BOD}$ and algae. The aluminum polychloride was appropriate to significantly reduce turbidity and $\mathrm{OSH}$ in a $92 \%$, it also presented the best economic viability in comparison to the other treatments; In regard to the decrease of total phosphorus moringa and cardon natural coagulants showed effective results, leading to a removal level of $90.35 \%$. It was concluded that the coagulants assessed showed a removal potential of the analyzed parameters, the results supported the Moringa oleifera as a possible natural alternative in the purification of water, because it guaranteed not only the success of the clotting process, but also the absence of residues.

Key-words: residual water, effluent, nutrients, eutrophication, test of jars.

\section{Resumo}

Avaliou-se o desenvolvimento de dois coagulantes químicos: sulfato de alumínio e policloreto de alumínio (PAC), e dois naturais: moringa (Moringa oleifera) e cardón guajiro (Lemaireoreusgriseus) na remoção de nutrientes e sedimentos dos efluentes da planta de tratamento das aguas residuis constituida por lagoas de estabilização. Salguero localizado no município de Valledupar, Cesar, Colômbia. Os testes foram realizados em amostras de água do rio César contido no sistema de tratamento de efluentes. Turbidez variou de 25 NTU a 67 NTU Durante o tempo de desenvolvimento da pesquisa verificou-se que o sulfato de alumínio, apesar de não atingir a turbidez exigido pela lei colombiana (Decreto 3930/2010, Artigo $\mathrm{N}^{\circ} 72$ Turbidez $\geq 5 \mathrm{NTU}$ ) é um coagulante que remove mais de $80 \%$ dos parâmetros $\mathrm{COD}, \mathrm{BOD}$ e algas. alumíniopolivinil é adequado para reduzir significativamente a turbidez e TSS em 92\%, também apresentou a melhor viabilidade econômica dos tratamentos; e para a redução do fósforo total resultaram efetivos os tratamentos com coagulantes naturais Moringa e Cardón, os quais apresentaram uma remoção $90,35 \%$. Concluiu-se que os coagulantes avaliados apresentam potencial de remoção dos parâmetros analisados, os resultados apoiam a Moringa oleifera como uma possível alternativa natural na purificação de água, uma vez que garante não só alcançar com sucesso o processo de coagulação, mas também, a ausência de resíduos.

Palavras-chave: águas residuais, efluentes, nutrientes, eutrofização, prova de jarras.

\section{Introducción}

Los efluentes de los sistemas de tratamientos de aguas residuales, presentan concentraciones de nutrientes tales como fosforo y nitrógeno, componentes causantes de la eutrofización en las corrientes de agua. Como sistemas de pos-tratamientos de nutrientes encontramos la coagulación que es un proceso común en el tratamiento de aguas aplicado para la desestabilización de impurezas

coloidales y disueltas, produciendo agregados de flóculos grandes que pueden ser removidos por subsiguientes procesos de filtración/clarificación (Gao, Hahn, H. \& Hoffmann, 2002). Este proceso se lleva a cabo agregando coagulantes, los cuales pueden ser clasificados como inorgánicos, polímeros orgánicos sintéticos y naturales (Okuda et al., 2001). 
Las sales de aluminio son los coagulantes más ampliamente utilizados en el tratamiento del agua, el cual ha demostrado ser un buen coagulante en lo que a remoción de turbidez y color se refiere (CEPIS 1983). Sin embargo, el uso de las sales de aluminio debe ser controlado ya que podrían dejar en el agua tratada cierta cantidad de aluminio residual (Romero, 1998). El aluminio residual presente en las aguas puede ser peligroso para la salud ya que está relacionado con el mal de Alzheimer (Stauber et al., 1999), producción de grandes volúmenes de lodo, reacción con la alcalinidad natural presentes en el agua y la baja eficiencia en la coagulación de aguas frías. Desventajas similares se han reportado para las sales de hierro y los polímeros sintéticos (Ndabigengesere, Narasiah \& Talbot, 1995).

En función de lo señalado, se hace necesario evaluar coagulantes y floculantes más efectivos, menos perjudiciales y ambientalmente inocuos para remover la turbidez de las aguas, con la finalidad de sustituir total o parcialmente las sales de hierro y aluminio y los polímeros orgánicos sintéticos. En este sentido los coagulantes naturales constituyen una alternativa viable porque son usualmente más seguros para la salud.

Recientemente, en Colombia se han desarrollado investigaciones con coagulantes naturales entre ellos el cardón guajiro (L. griseus), obteniendo resultados satisfactorios en el proceso de coagulación (Fonseca \& Vásquez, 1984). En países como Venezuela se ha estudiado el exudado gomoso producido por Samanea Saman (González et al., 2006), la corteza del cactus lefaría (Martínez et al., 2003) y las semillas, sin grasa, de M. oleifera (Mendoza, Fernández, Ettiene \& Díaz, 2000), los cuales han demostrado ser eficientes en tratamientos de agua.

La M. oleífera es una planta tropical perteneciente la familia Moringaceae. Es originaria del noroeste de la India, y es conocida en Brasil como quiabo de quina y Lirio testigo (López \& Silva, 2004). El coagulante es declaradamente activo, seguro y barato (Castro \& Silva, 2004; Ghebremichael et al., 2005, Ndabigengesere et al., 1995), siendo posible su amplia utilización en el tratamiento del agua, lo que trae beneficios económicos para los países productores, además de constituirse en una alternativa ambientalmente correcta. (Caldera et al. 2007), evaluaron la eficiencia de las semillas de $M$. oleífera como coagulante natural en la potabilización de aguas sintéticas con valores de turbidez de 75 y 150 NTU. Por otra parte, Muyibi \& Okuofu (1995), evaluaron el efecto del aceite extraído de las semillas de $M$. oleífera sobre la coagulación de aguas, con turbidez inicial entre 56 y 451 NTU, reportando valores de remoción superiores al $87 \%$.

Los cactus contienen una sustancia que al contacto con el agua toma una consistencia coloidal. Basado en esta propiedad que presenta el cactus se hicieron estudios de la acción coagulante en la Universidad del Atlántico, BarraquiIla, Colombia por los Ingenieros químicos Fonseca \& Vásquez (1984). En esta investigación se determinó que el cardón guajiro (L. griseus), como coagulante puede llegar a representar una alternativa para ser usado en plantas de tratamiento de aguas, para turbiedades comprendidas entre 30 y 190 NTU el cardón es capaz de reducir la turbiedad en aguas decantadas a valores comprendidos entre 5 y 10 NTU, además en este estudio se estableció, a diferencia de los coagulantes convencionales que forman los flocs en las etapas de mezcla lenta, que con el cardón guajiro (L. griseus) los flocs aparecen instantáneamente en la etapa de mezcla rápida; esto presenta una gran ventaja pues disminuye el tiempo de residencia y con ello se aumenta el volumen de agua tratada, por otro lado se implantó que el cardón produce los mejores efectos de coagulación cuando la solución dosificadora tiene una concentración comprendida entre el $2 \%$ y el $5 \%$, y que los factores o agentes químicos que intervienen en la coagulación tales como el pH, la pre cloración, la presencia de otros coagulantes y la alcalinidad no modifican el poder coagulante del cardón. 
La planta de tratamiento de aguas residuales Estación Salguero ubicada en Valledupar, Colombia, descarga su efluente en la fuente hídrica del río Cesar, el cual ha presentado valores máximos de turbidez desde 25 hasta 67 NTU. En esta investigación se evaluó la eficiencia de las semillas de moringa y el cardón guajiro con los coagulantes químicos sulfato de aluminio y policloruro de aluminio PAC en la remoción de nutrientes en efluente de agua residual.

\section{Materiales y métodos}

Las muestras de agua residual (40I) se colectaron en el efluente del sistema de tratamiento de aguas residuales de la planta de tratamiento Salguero, antes de ser vertidas a la fuente hídrica del rio Cesar. La planta se encuentra ubicada en el sureste del municipio de Valledupar, departamento de Cesar, Colombia. Las muestras colectadas se trasladaron al laboratorio agroindustrial CIDI de la Universidad Popular del Cesar y se almacenaron para su posterior caracterización.

Preparación de la solución de los coagulantes naturales cardón guajiro (L. griseus) y M. oleífera Las plantas de cardón guajiro (L. griseus), se recolectaron en estribaciones de San Juan del Cesar en el departamento de La Guajira, Colombia. La parte aprovechable del cardón es la pulpa del tallo o sea la sección comprendida entre la corteza y la medula tubular leñosa. La pulpa fue separada de las espinas, la corteza y de la medula tubular leñosa y ésta fue sometida a trituración a través de un mortero, hasta obtener el producto coagulante mediante la filtración a la que fue sometido.

Las semillas de la Moringa oleífera, fueron suministradas por la empresa COLMORINGA en Bogotá D.C., la especie se reproduce en abundancia en el departamento de Tolima, Colombia. Se trasladaron al laboratorio aproximadamente 300 semillas secas sin uniformidad de tamaño, provenientes de varias plantas. Posteriormente se extrajeron los cotiledones de las semillas y licuaron (licuadora tradicional) con $2.5 \mathrm{mg}$ con 100 $\mathrm{ml}$ de agua destilada; la solución se filtró y se utilizó como coagulante, obteniendo una solución de color testigo amarillento, de aspecto bastante grasoso y olor muy particular.

Preparación de la solución de los coagulantes químicos policloruro de aluminio (PAC) y sulfato de aluminio $A L_{2}\left(\mathrm{SO}_{4}\right)^{3}$

Los coagulantes químicos sulfato de aluminio y poli-cloruro de aluminio (PAC), se trabajaron con la concentración comercial para tratar aguas residuales.

\section{Proceso de coagulación}

La evaluación de la coagulación se llevó a cabo utilizando un aparato de prueba de jarro; se agregó 1 de agua residual a cada uno de los 6 vasos, de 2 l cada uno. Los recipientes se colocaron por debajo de los agitadores y se les proporcionó una velocidad de 100 rpm; dejando uno de estos como patrón. Posteriormente, se procedió a agregar los coagulantes naturales y químicos, al iniciar el mezclado rápido (100 rpm, 1min); las diferentes dosis de coagulantes químicos y naturales respectivamente $\left(45,50,55,60,65 \mathrm{mg} \mathrm{l}^{-1}\right.$; y dosis mayores para los naturales $600,650,700$, $750,800 \mathrm{mg} \mathrm{l}^{-1}$ ), las dosis se agregaron en cada vaso usando una pipeta para el mezclado rápido y luego se procedió al mezclado lento, para finalizar con la fase de sedimentación.

Con los coagulantes químicos se manejó un mezclado lento (40rpm), el agua se dejó flocular por 15 min para finalizar en la fase de sedimentación (10min). Los coagulantes naturales como M. oleífera se manejaron con un mezclado lento (30rpm), para finalizar con la fase de sedimentación (60 min); con el coagulante obtenido del cardón guajiro (L. griseus) se manejó una mezcla lenta de (60rpm) y la sedimentación (60 min).

\section{Parámetros analizados}

La turbidez, pH, temperatura, fosforo total, Demanda química de oxígeno, Demanda biológica de oxígeno, algas y sólidos suspendidos totales, se 
determinaron según lo establecido en los métodos estándar (APHA, AWWA, WEF, 1998). La turbidez se midió en el turbidímetro digital Scientic, Inc modelo HACH 2100 AN. Para medir la temperatura se hizo con lectura directa del termómetro, el pH con lectura directa $\mathrm{pHmetro}$; el fosforo total se determinó con espectrofotometría, y la Demanda Química de Oxigeno (DQO): se realizó mediante el método de reflujo cerrado (colorimétrico y titulométrico). SM 5220 D, finalmente la Demanda Biológica de Oxigeno (DBO): Dilución e incubación 5 días. SM $5210 \mathrm{~B}$.

\section{Resultados}

Caracterización físico-química del efluente de la planta de aguas residuales "El Salguero"

Los parámetros fisicoquímicos iniciales de las muestras de agua se muestran en la Tabla 1. Las concentraciones de algunos parámetros como los sólidos suspendidos totales arrojan resultados de $76,33 \mathrm{mg} \mathrm{l}^{-1}$ que comparados con los resultados del afluente, datos suministrados por la empresa de servicios públicos EMDUPAR (2011), muestran que sólo se alcanza a remover el $38.44 \%$ durante todo el tratamiento desde la entrada del sistema de tratamiento de aguas residuales hasta la llegada del agua al cuerpo receptor, valores que se encuentran por encima del valor máximo admisible permitido por la norma (Decreto 3930/2010 Art.72. SST $\geq 50 \%$ en carga para usuario existente). Sin embargo la DQO y DBO muestran remociones de $60 \%$ cumpliendo con la normatividad Colombiana de vertimientos (Decreto 3930/2010 art 72 DQO $\geq 30 \%$ en carga para usuario existente), pero es necesario la eliminación de estos parámetros porque afectan significativamente el cuerpo de agua; por otro lado las concentraciones de fosforo total en el efluente tratado fueron de hasta $33,66 \mathrm{mg}$ $\mathrm{I}^{-1}$ permitiendo gran turbiedad y alta concentración de algas.

Tabla 1. Parámetros fisicoquímicos iniciales del efluente de la planta de tratamiento "El Salguero"

\begin{tabular}{lcc}
\hline Parámetros Fisicoquímicos & Unidad de Expresión & Efluente de Estación Salguero \\
\hline Temperatura & ${ }^{\circ} \mathrm{C}$ & 24.6 \\
$\mathrm{pH}$ & $\mathrm{UpH}$ & 7.01 \\
Turbidez & $\mathrm{NTU}$ & 42.9 \\
SST & $\mathrm{mg} \mathrm{l}^{-1}$ & 76.33 \\
DQO & $\mathrm{mg} \mathrm{l}^{-1}$ & 194 \\
DBO & $\mathrm{mg} \mathrm{l}^{-1}$ & 88 \\
\hline Fosforo Total & $\mathrm{mg} \mathrm{l}^{-1}$ & 33.66 \\
Algas & $\mathrm{mg} \mathrm{l}^{-1}$ & 1.10376 \\
\hline
\end{tabular}

Dosis óptima de sulfato de aluminio, policloruro de aluminio (PAC), M. oleífera y cardón guajiro (L. griseus)

Los resultados de las pruebas de jarras de cada uno de los coagulantes muestra las dosis óptima de los diferentes tratamientos donde se obtuvieron las turbiedades más bajas, teniendo en cuenta que la turbidez del efluente de la planta de tratamiento El Salguero varía desde 25 a 67 NTU. Con los coagulantes químicos sulfato de aluminio y policloruro de aluminio se manejaron dosificaciones a partir de $45 \mathrm{mg} \mathrm{l}^{-1}$ a $65 \mathrm{mg} \mathrm{l}^{-1}$, 
obteniéndose en el caso del sulfato de aluminio una dosis de $55 \mathrm{mg} \mathrm{l}^{-1}$, una turbidez de 6,35 NTU. Para el policloruro de aluminio con una dosis de $50 \mathrm{mg} \mathrm{l}^{-1}$ se obtuvo una turbidez bastante baja de 0,333 NTU (Figura 1) lo que corrobora una vez más que el policloruro de aluminio presenta buen rendimiento como coagulante primario logrando cumplir con la Normativa Colombiana según lo descrito en el Articulo N72 Turbidez $\geq 5$ NTU (Decreto 3930 del Ministerio de Ambiente, Vivienda y Desarrollo
Territorial 2010). Con las dosis de coagulantes químicos se obtuvieron sobrenadantes clarificados, removiéndose el color aparente y observándose en el fondo de cada una de las jarras una capa de color verde; que se le atribuye a la sedimentación de algas y a los sólidos en suspensión. El mecanismo de adsorción neutralización de cargas predomina en las bajas dosificaciones de sulfato de aluminio, mientras que el mecanismo de barredura predomina en mayores dosificaciones de coagulante.

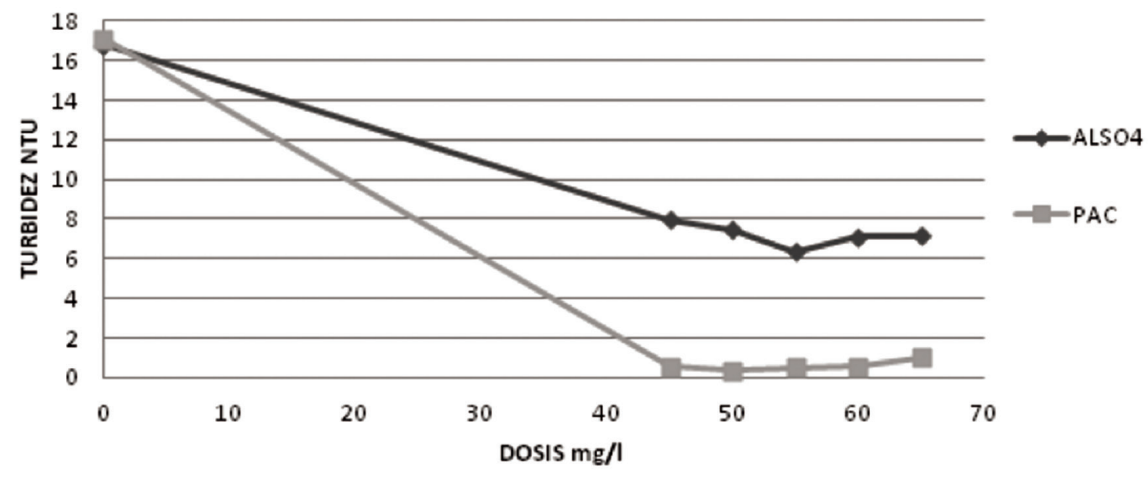

Figura 1. Dosis óptima de sulfato de aluminio y policloruro de aluminio

En relación a los coagulantes naturales $M$. oleífera y cardón guajiro (Figura 2), se manejaron dosis mayores a diferencia de los coagulantes químicos. En el caso de M. oleífera se utilizaron dosis de $600 \mathrm{mg} \mathrm{l}^{-1}$ a $800 \mathrm{mg} \mathrm{l}^{-1}$, obteniéndose la turbidez más baja de 6.56 NTU con una dosis de $700 \mathrm{mg} \mathrm{l}^{-1}$; y para el cardón guajiro se utilizaron dosis desde $1200 \mathrm{mg} \mathrm{l}^{-1}$ hasta $1800 \mathrm{mg} \mathrm{l}^{-1}$ siendo la dosis óptima de $1500 \mathrm{mg} \mathrm{l}^{-1}$ alcanzando una turbidez de 10,6 NTU. La efectividad de los coagulantes $M$. oleífera y sulfato de aluminio se comparó para turbiedades iniciales de 7, 11, 15, 20, 29, y 49 NTU. Para cada coagulante se aplicó la dosis óptima.

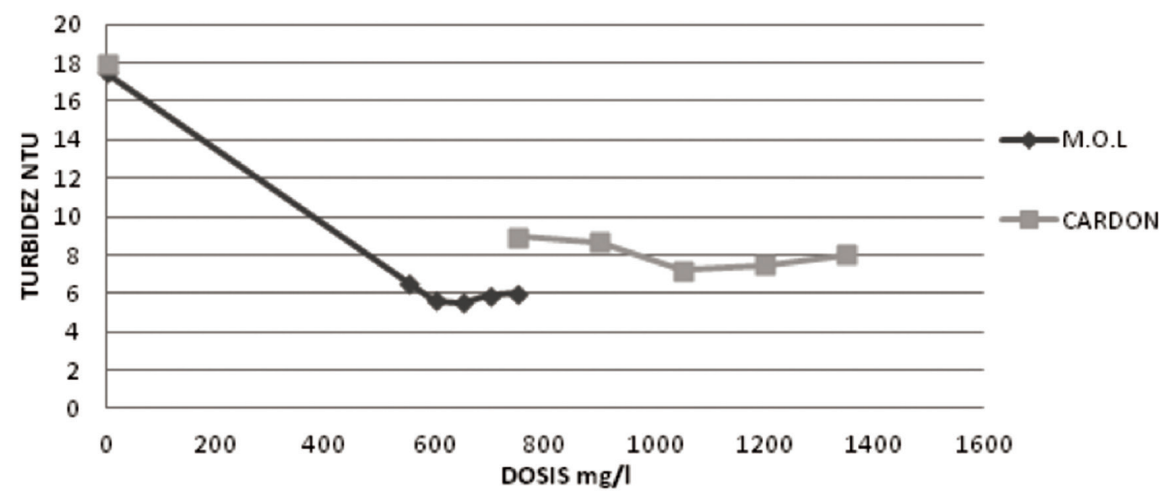

Figura 2. Dosis óptima de Moringa oleífera y cardón guajiro 
Al evaluar a escala laboratorio la remoción de SST se obtuvo que en todos los tratamientos existieron significativas remociones, incluso en el testigo que no tenía ningún tipo de tratamiento se lograron apreciables valores de remoción; situación que podría obedecer a que la reducción de sólidos suspendidos se rige por procesos físicos como sedimentación, decantación, y también filtración, sin embargo los mayores porcentajes de remoción promedio fueron obtenidos en los tratamientos con el policloruro de aluminio (PAC) con un $91,16 \%$, y siguió el tratamiento con sulfato de aluminio $\left(\mathrm{ALSO}_{4}\right)$ con un $84,12 \%$, por último se presentan los coagulantes naturales, con $M$. Oleífera se logró un porcentaje de remoción de $73,20 \%$, seguidamente el tratamiento con cardón guajiro
(L. griseus) con $65,36 \%$ y con el menor valor de remoción los testigos con un 43,10\%; (Figura 3). Por todo esto se puede afirmar que el tratamiento con el policloruro de aluminio PAC fue el más eficiente, puesto que posee excelentes porcentajes de remoción y cumple ampliamente con la Normativa Colombiana en relación al Art.72. SS $\geq 50 \%$ en carga para usuario existente (Decreto $3930 \mathrm{del}$ Ministerio de Ambiente, Vivienda y Desarrollo Territorial 2010). Es importante destacar que el criterio para seleccionar la concentración óptima del coagulante sulfato de aluminio se corresponde a una menor concentración que permita obtener una menor turbidez y menor residual de aluminio, ya que la ingesta en altas concentraciones de este metal es perjudicial para la salud.

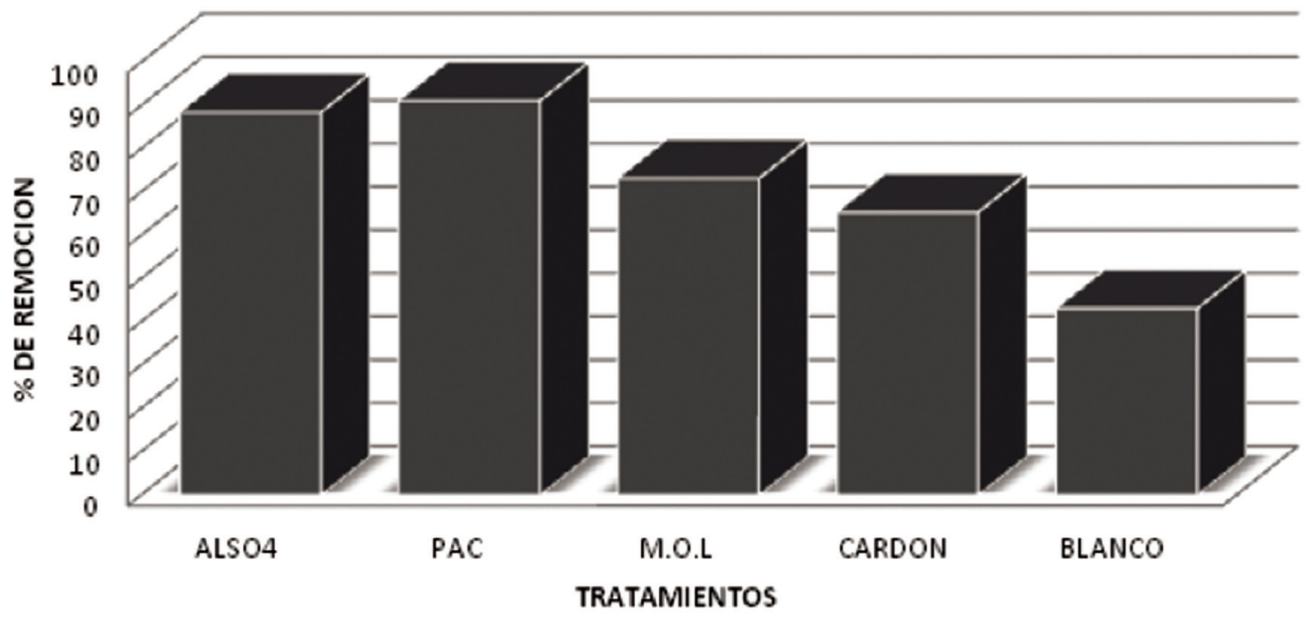

Figura 3. Porcentaje de remoción de sólidos suspendidos totales por cada tratamiento

En promedio, el rango de $\mathrm{pH}$ entre los tratamientos con policloruro de aluminio PAC, Moringa Oleífera y cardón guajiro ( $L$. griseus) estuvo en 7,01(Ajustándose al Decreto 3930/2010 Art.72. pH de 5 a 9 unidades), esto valores de $\mathrm{pH}$ contrastan con lo establecido por Ndabigengesere et al. (1995) donde señalan que el proceso de coagulación no se ve alterado por el pH inicial del medio; dicho de otro modo, el proceso de coagulación es independiente del $\mathrm{pH}$. Este es un dato muy interesante desde el punto de vista para su aplicación directa en una planta de tratamientos de aguas, ya que no se debe corregir el $\mathrm{pH}$ del agua al ingreso del proceso. El valor promedio de $\mathrm{pH}$ obtenido con el tratamiento de sulfato de aluminio se encontró en 4,8 (Figura 4.); valor que no es recomendable para la buena salud de los cuerpos de agua ya que tener $\mathrm{pH}$ bajos puede alterar la flora y la fauna nativa y además puede afectar la posible utilización de estas aguas (cuerpo receptor) para las actividades antrópicas. 


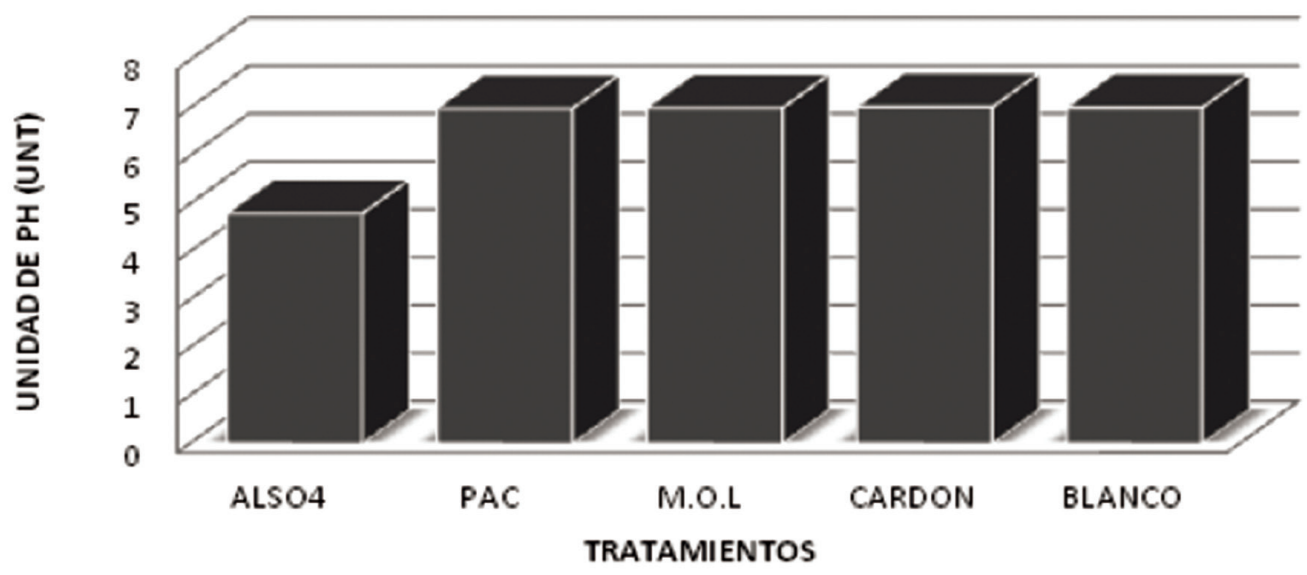

Figura 4. $\mathrm{pH}$ promedio por cada tratamiento.

Los resultados obtenidos en el estudio de la Demanda Química de Oxigeno mostraron diferencias significativas entre los tratamientos, es así como al analizar la remoción de este parámetro se observaron mayores remociones entre los tratamientos con los coagulantes químicos sulfato de aluminio con $84,71 \%$ y policloruro de aluminio con $81,91 \%$, y el coagulante natural cardón guajiro ( $L$. griseus) con una remoción de $80,24 \%$ (Figura 5) logrando cumplir ampliamente con la norma ambiental según el art 72 DQO $\geq 30 \%$ en carga para usuario existente (Decreto 3930 del Ministerio de Ambiente, Vivienda y Desarrollo Territorial 2010).

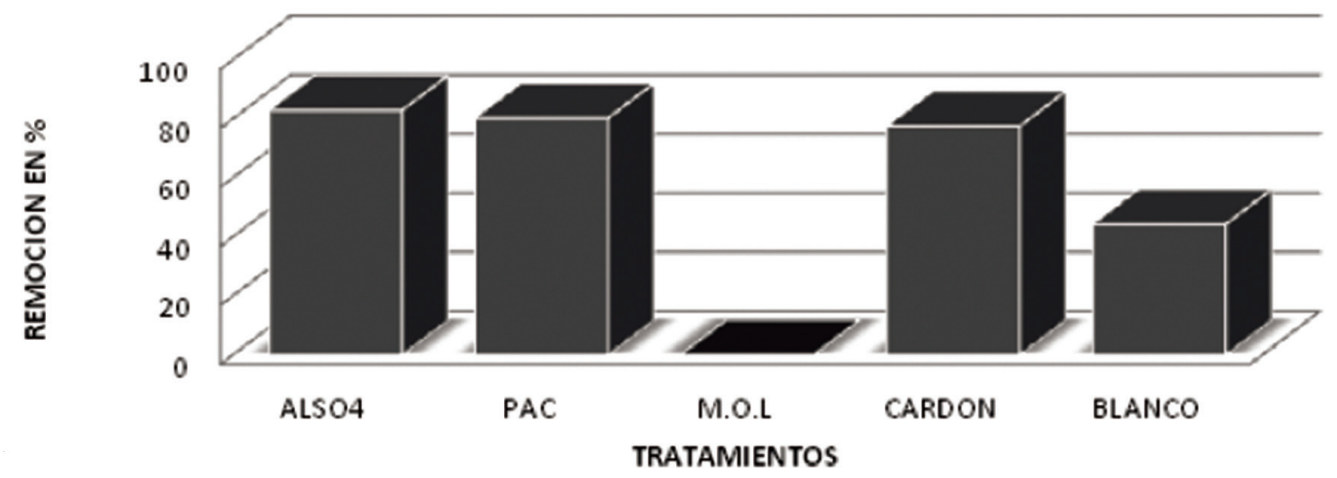

Figura 5. Porcentaje de remoción de DQO por cada tratamiento

En la DBO se puede observar en la Figura 6 que los mayores valores de remoción fueron los obtenidos con los tratamientos de sulfato de aluminio (ALSO4) y policloruro de aluminio (PAC) en $69,95 \%$ y $64,27 \%$ respectivamente. Con respecto al tratamiento con cardón guajiro ( $L$. griseus) se obtuvo una remoción de DBO de $62,63 \%$ e incluso en la muestra sin tratamiento es decir el testigo existió una mínima remoción de 25,51\% lo que indica que estos tratamientos cumplen ampliamente con la normatividad colombiana (Decreto 3930/2010 art. 72 DBO $\geq 30 \%$ en carga, para usuario existente); a pesar de que la $M$. oleífera es un coagulante de origen natural al igual que el cardón guajiro ( $L$. griseus) se pudo apreciar que la concentración de DBO aumentó esto es debido que la DBO contenida en la solución de M. oleifera queda suspendida en la muestra y no se sedimenta con el floc formado, este aumento es un 
factor negativo para los procesos de descontaminación de una planta de tratamiento de aguas residuales y además puede afectar la utilización de la M. oleifera como coagulante.

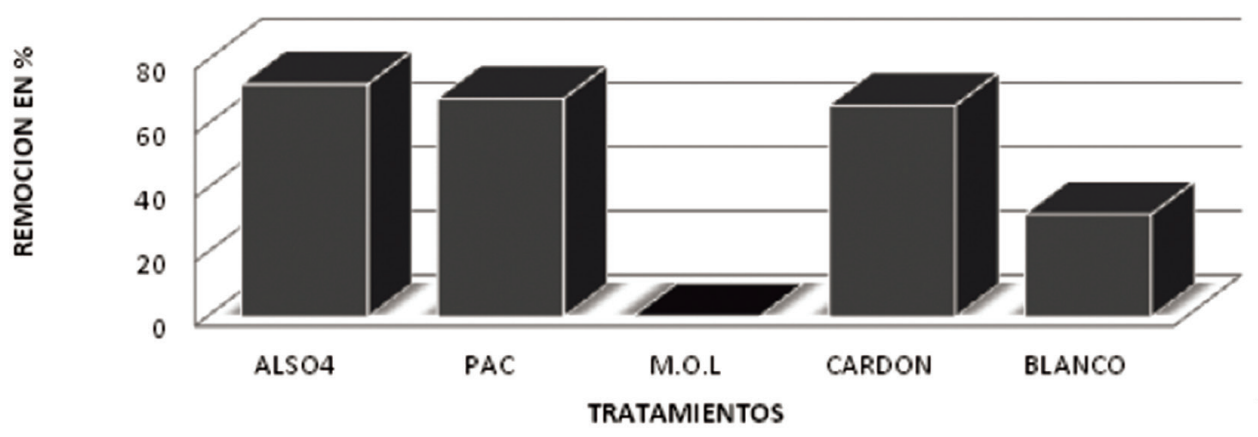

Figura 6. Porcentaje de remoción de DBO en cada tratamiento

\section{Discusión y conclusiones}

En los diferentes tratamientos manejados en la prueba de jarras se obtuvieron las turbiedades más bajas (Tabla 2), teniendo en cuenta que la turbidez del efluente de la planta El Salguero varía desde 25 a 67 NTU, este registro es similar a los valores de turbidez de 7 y 49 NTU manejados por Mendoza et al., (2000) y los registros de 75 y 150 NTU aportados por Caldera et al., (2007) para la potabilización de aguas crudas sintéticas.

Ledo et al. (2009) identificaron que la mejor eficiencia de remoción por parte del sulfato de aluminio fue observada en la dosificación de 15 $\mathrm{mg} \mathrm{l}^{-1}$ correspondiendo a un porcentual de remoción del $84 \%$ y en dosificaciones por encima de
$80 \mathrm{mg} \mathrm{l}^{-1}$, se verificó la formación de copos mayores y una mejora en la remoción de turbiedad. En esta investigación el sulfato de aluminio con la dosis de $55 \mathrm{mg} \mathrm{l}^{-1}$, obtuvo una turbidez de 6,35 NTU. Para el policloruro de aluminio con una dosis de $50 \mathrm{mg} \mathrm{l}^{-1}$ se obtuvo una turbidez bastante baja de 0,333 NTU. Ndabigengesereet al. (1995), compararon el rendimiento de los coagulantes sulfato de aluminio y $M$. oleífera y verificaron que los dos coagulantes presentaron dosis óptimas próximas a $50 \mathrm{mg} \mathrm{l}^{-1}$. Por otra parte, según Schwarz para aguas con turbiedad debajo de 50 NTU, la banda de dosis de semillas empleada es entre 10 y 50 $\mathrm{mg} \mathrm{L}^{-1}$. (Schwarz, 2000). Algunos investigadores presentan resultados similares, pudiéndose citar: Lacerda (1997), Pavanelli (2001), Teixeira (2003), entre otros.

Tabla 2. Análisis fisicoquímico y microbiológico del agua residual tratada con coagulantes

\begin{tabular}{lcccccccccc}
\hline Parámetro & $\mathbf{A l}_{\mathbf{2}}\left(\mathbf{S O}_{\mathbf{4}}\right)_{\mathbf{3}}$ & \%R & PAC & $\% \mathbf{R}$ & M.O.L & $\% \mathbf{R}$ & Cardón & $\% \mathbf{R}$ & Testigo & $\% \mathbf{R}$ \\
\hline DQO & 29,66 & 84,71 & 35,1 & 81,91 & 197,98 & - & 38,34 & 80,24 & 93,33 & 51,89 \\
DBO & 26,44 & 69,95 & 31,44 & 64,27 & 110,25 & - & 32,89 & 62,63 & 65,55 & 25,51 \\
Fosforo & 10,82 & 67,86 & 8,121 & 75,87 & 4,96 & 85,26 & 3,18 & 90,55 & 18,55 & 44,89 \\
Algas & 0,0262 & 97,63 & 0,132 & 88,08 & 0,1603 & 85,48 & 0,19113 & 82,68 & 0,76877 & 30,35 \\
SST & 12,12 & 84,12 & 6,75 & 91,16 & 20,46 & 73,20 & 26,44 & 65,36 & 43,43 & 43,10 \\
pH & 4,8 & & 6,29 & & 6,96 & & 7 & & 7,01 & \\
\hline
\end{tabular}


Al evaluar a escala laboratorio el efecto de los coagulantes químicos y naturales en la eliminación de fosforo, se pudo apreciar que existieron diferencias significativas entre los tratamientos con coagulantes naturales vs tratamientos con coagulantes químicos. Los tratamientos con coagulantes naturales lograron los mayores porcentajes de remoción: con
M. oleífera $85,26 \%$ y cardón guajiro (L. griseus) $90,55 \%$. Posteriormente se encuentran los tratamientos con coagulantes químicos: policloruro de aluminio y sulfato de aluminio los cuales obtuvieron remociones de $77,87 \%$ y $67,86 \%$, respectivamente, y los testigos $44.89 \%$ manteniendo los menores valores de eliminación de este parámetro (Figura 7).

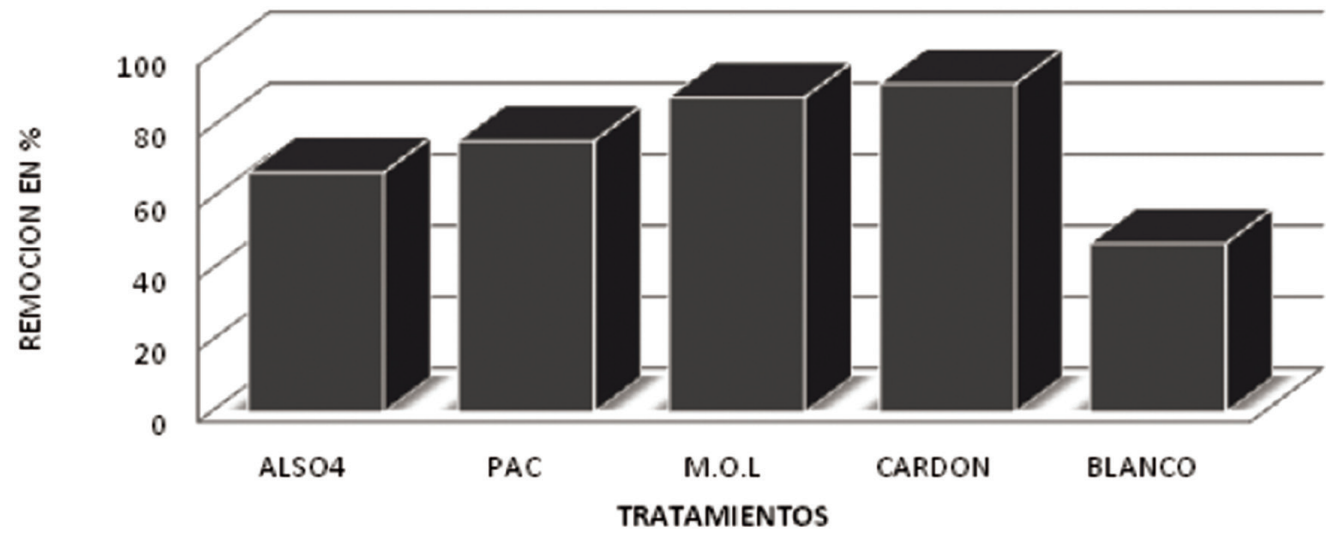

Figura 7. Porcentaje de remoción de fosforo total por tratamiento.

Las dosis aplicadas de ambos coagulantes fueron similares para cada valor de turbidez inicial a excepción de la muestra con turbidez inicial de 20 NTU que requirió $35 \mathrm{mg} \mathrm{l}^{-1}$ de sulfato de aluminio y $15 \mathrm{mg}$ $\mathrm{I}^{-1}$ de M. oleífera (Mendoza et al., 2000). Otros investigadores han reportado que, usando diferentes dosis de $M$. oleífera como coagulante en la potabilización de aguas, con valores bajos, moderados y altos de turbidez inicial, se obtienen porcentajes de remoción superiores al 50\% (Muyibi \& Okuofu, 1995).

A diferencia de los coagulantes químicos que forman los flocs en la etapa de mezcla lenta, en los coagulantes naturales en estudio los flóculos aparecen en la mezcla rápida; esto representa una gran ventaja pues disminuye el tiempo de residencia y con ello se aumenta el volumen de agua tratada; estos flóculos resultan de buena consistencia, de ahí que los gradientes altos de velocidad de floculación, lejos de desintegrarlos favorecen su aglutinación (Fonseca \& Vásquez, 1984).

La efectividad en la remoción de turbidez de ambos coagulantes es equiparable, sin embargo, la
M. Oleífera pudiera presentar ventajas sobre el sulfato de aluminio ya que estudios realizados han demostrado que al utilizar $M$. oleífera no quedan residuos tóxicos en el agua tratada que pudieran afectar el organismo humano, dado su origen vegetal (Jahn 1988). Sin embargo el tratamiento con $M$. oleífera no presenta ningún tipo de remoción por el contrario se aumenta la concentración de la demanda química de oxígeno en la muestra, lo que revela que este tipo de coagulante contiene DQO que al adicionarla a la muestra queda suspendida y no se sedimenta. Carpinteyro (2011), utilizó polímeros naturales como las gomas de algarrobo, semilla de mezquite y mucilago de nopal como coagulantes-floculantes para el tratamiento de aguas residuales tanto municipales como industriales, alcanzando remociones de DQO entre 42 y $51 \%$, mientras que en esta investigación con el cardón guajiro se obtuvieron valores del $80,24 \%$.

Al comparar los tratamiento de macrofita Lemna ssp y Eichornnia caprisses con los coagulantes naturales y químicos, se observa que este tipo de tratamiento biológico tiene una menor eficiencia 
de eliminación de fosforo que el trabajado con los coagulantes, dado que obtuvo un porcentaje promedio de $62,72 \%$, además el tiempo de remoción por coagulación es mucho menor que el tiempo que se necesita con la utilización de MacrofitasIemna ssp y Eichornnia caprisses. De acuerdo con la investigación la eliminación de algas con los tratamientos químicos resultó altamente efectiva (Figura 8); la mayor remoción de algas se obtuvo con el tratamiento de sulfato de aluminio en un $97.63 \%$ seguido del tratamiento con policloruro de aluminio en un $88.08 \%$, posteriormente se encuentra los tratamientos con los coagulantes naturales con una remoción de $85,48 \%$ para la $M$. oleífera y $82.68 \%$ para el cardón guajiro ( $L$. griseus) valores muy importantes porque con la eliminación casi completa de este parámetro se excluye la existencia del color y la turbidez en el agua, además se atribuye que esta ausencia de masa de algas permite establecer que no hay presencia de fosforo en el agua. La mayor remoción de algas se obtuvo con el coagulante de sulfato de aluminio, aunque no existe correlación entre eliminación de algas y la turbiedad final obtenida con la aplicación de este coagulante, coincide con lo hallado por States, Tomko, Scheuring \& Casson (2002); quienes afirman que la turbiedad no siempre es un indicador confiable de la remoción de algas ya que, aun cuando altos niveles de turbiedad pueden ser un indicio del pasaje de algas, valores bajos no proveen absoluta confianza sobre la eliminación de las mismas. Estos altos porcentajes de eliminación de algas, que indican una disminución notoria de la carga de nutrientes, contribuirían al éxito en el proceso de descontaminación del agua residual. Como se puede observar en el Figura 8 el testigo, que no contiene ningún tipo de tratamiento, presenta también un valor mínimo de remoción de 30,35\%, se considera que esta pequeña remoción se da por procesos físicos (sedimentación, decantación, y también filtración).

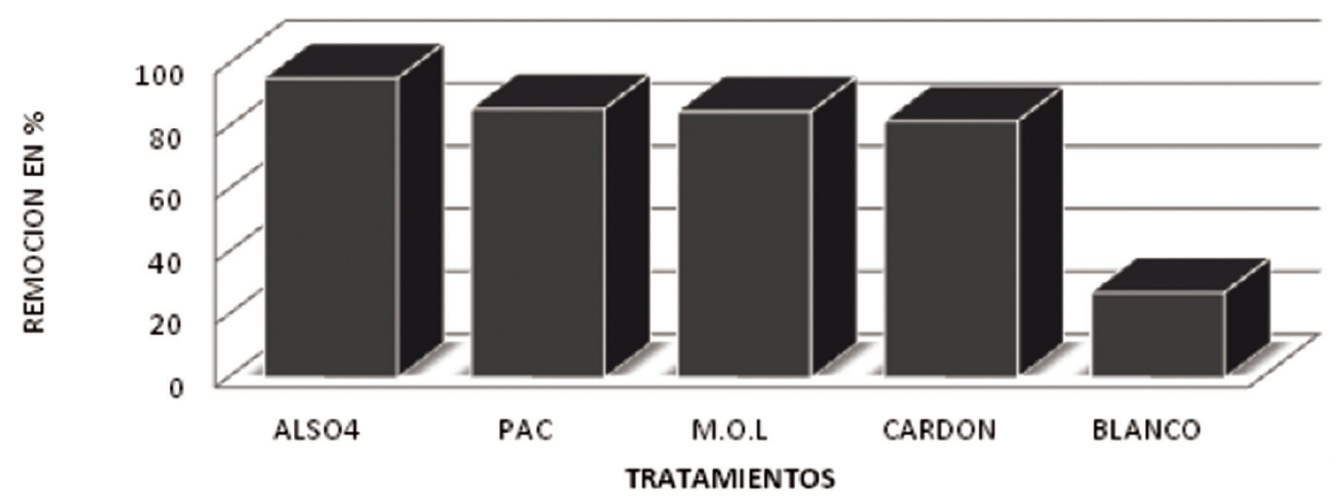

Figura 8. Porcentaje de remoción de algas por tratamiento

Estos resultados avalan a la $M$. oleifera como una posible alternativa natural en la potabilización de las aguas, ya que garantiza no sólo lograr con éxito el proceso de coagulación, sino también, la ausencia de residuos. Sin embargo, al hacer un análisis de costo se encontró que los costos de tratamiento con $M$. oleífera son excesivamente altos en comparación con los del sulfato de aluminio. Estos costos tan altos se deben al empleo de éter de petróleo y alcohol isopropílico para la eliminación de la grasa presente en las semillas. El costo que genera la utilización de papel de filtro en la etapa de extracción de la grasa resultó insignificante. Todas estas consideraciones validan la potencialidad de esta especie vegetal en el tratamiento de aguas, razones por las cuales se debe seguir investigando, especialmente en lo relacionado al establecimiento de un procedimiento de extracción de la grasa más económico (Mendoza et al., 2000). 


\section{Agradecimientos}

Los autores agradecen al Programa de Ingeniería Ambiental y Sanitaria de la Universidad Popular del Cesar en el municipio de Valledupar, Colombia, por la financiación del proyecto investigación realizado y por el apoyo en el desarrollo exitoso del mismo, tanto en la fase de campo como en la de laboratorio, ofreciendo sus instalaciones.

\section{Literatura citada}

1. Arboleda, J (1997). Teoría, Diseño y control de los procesos de clarificación del agua, Centro Panamericano de Ingeniería sanitaria y ciencias del ambiente. Lima Perú.

2. APHA/AWWA/WEF (1998) Standard methods for the examination of water and wastewater. 20th edition. American Public Health Association / American Water Works Association / Water Environment Federation, Washington, DC.

3. Caldera, Y., Mendoza, I.. Briseño, L., García, J. \& Fuentes, L. (2007). Eficiencia de las semillas de M. oleífera como coagulante alternativo en la potabilización del agua. Boletín del centro de investigaciones biológicas volumen 41, no. 2: 244-254 Universidad del Zulia, Maracaibo, Venezuela.

4. Carpinteyro Urbán, Sandra Lucero (2011). Tratamiento de Aguas Residuales Empleando Polímeros Naturales y Biodegradabilidad de los Lodos Generados. Instituto Politécnico Nacional Unidad Profesional Interdisciplinaria de Biotecnologia. México, D.F. Junio.

5. Castro, F.J.F \& Silva, F.J.A. (2004). Moringa ole ífera na Melhoria da Qualidade de Efluentes de UASB e de Lagoa de Maturação-Remoção de Cor e Turbidez. In: XI SILUBESA - Simposio Luso-Brasileiro de Engenharia Sanitária e Ambiental, Natal/RN.

6. Centro Panamericano de ingeniería sanitaria y ciencias del ambiente CEPIS (1983). Teoría, diseño y control de los procesos de clarificación del agua, Lima (Perú), 85-623.

7. EMDUPAR, Empresa de Servicios Públicos de Valledupar. (2011). Estudio de Caracterización del Agua Residual Afluente y Efluente El Salguero.

8. Fonseca Gómez, Demóstenes \& Vásquez Espalza, Miguel. (1984). El cardón como coagulante en el tratamiento de agua. Universidad del Atlántico Barraquilla.

9. Gao, B., Hahn, H. \& Hoffmann, E. (2002). Evaluation of aluminum-silicate polymer composite as a coagulant for water treatment. Water Research 36: 3573-3581.

10. Ghebremichael, K.A. et al. (2005). A simple purification and activity assay of the coagulant protein from Moringa oleifera seed. Water Research: 39, 2338-2344 (2005).

11. González, G., Chávez, M., Mejias, D., Mas, M., Rubí, Y., Fernández, N \& León, G. (2006). Uso del exudado gomoso producido por Samanea saman en la potabilización de las aguas. Rev. Técnica Fac Ingeniería Univ. del Zulia 29: 14-22.

12. Jahn Saa. (1988). Using Moringa seeds as coagulants in developing countries. J Am Water Works Assoc;80:43-50

13. Lacerda, M.R.S. (1997). A Influência do pH de Coagulação e do Tempo de Floculação na Flotação por ar Dissolvido no Tratamento de Água com Baixa Turbidez e Presença de Algas. Tesis de Magister, Departamento de Engenharia Civil e Ambiental, UNB, Brasília, Brasil.

14. Ledo, P., Lima, G., Raquel, F.S., João, B.A. \& Duarte, M. (2009). Estudio Comparativo de Sulfato de Aluminio y Semillas de Moringa ole ífera para la Depuración de Aguas con Baja Turbiedad. V. 20 n.5: 3-12.

15. López, A. S. \& Silva, F. (2004). Tratamiento de Efluente de Tanque de Piscicultura com Moringa oleífera. In: XI SILUBESA - Simpósio Luso-Brasileiro de Engenharia Sanitária e Ambiental, Natal/RN (2004).

16. Martínez, D., Chávez, M., Díaz, A., Chacín, A. \& Fernández, N. (2003). Eficiencia del Cactus Lefaria para su uso como coagulante en la clarificación de las aguas. Rev. Técnica Fac. Ingeniería Univ. del Zulia 26: 27-33.

17. Mendoza, I., Fernández, N., Ettiene, G. \& Díaz, Y. (2000). Uso de la Moringa oleífera como coagulante en la potabilización de las aguas. Ciencia 8: 243-254.

18. Muyibi, S. \& Okuofu, C. (1995). Coagulation of low turbidity surface water with Moringa ole ifera seeds. Intern. J. Environ. Studies 48: 263-273.

19. Ndabigengesere A., Narasiah, K.S. \& Talbot, B.G. (1995). Active Agents and Mechanism of Coagulation of Turbid Waters Using Moringa ole iffera. Water Research: 29 (2), 703-710.

20. Ndabigengesere, A. \& Narasiah, K.S. (1998). Quality of Water Treated by Coagulation Using Moringa ole ifera Seeds. Water Research: 32(3), 781-791.

21. Okuda, T., A.U. Baes, W. Nishijima \& Okada, M. (2001). Coagulation mechanism of salt solution extracted active components in Moringa ole ífera seeds. Water Res 2001;35:830-4.

22. Okuda, T., A.U. Baes, W. Nishijima \& Okada, M. (2001). Isolation and characterization of coagulant extracted from Moringa ole iffera seed by salt solution. Water $R e$ 2001;35:405-10.

23. Okuda, T., A.U. Baes, W. Nishijima \& Okada, M. (2001b). Coagulation mechanism of salt solution- extracted active component in Moringa ole ífera seeds. Water Research: 35(3), 830-834 (2001b).

24. Okuda, T., A.U. Baes, W. Nishijima \& Okada, M. (2001a). Isolation and Characterization of Coagulant Extracted from Moringa ole ífera Seed by Salt Solution. Water Research: 35(2), 405-410 (2001a).

25. Pavanelli, G. (2001). Eficiência de Diferentes Tipos de Coagulantes na Coagulação, Floculação e Sedimentação de Água com Cor ou Turbidez Elevada. Tesis de Magister, Escola de Engenharia de São Carlos, USP, São Carlos, Brasil. 
26. Presidencia de la Republica de Colombia Decreto 3930 del Ministerio de Ambiente, Vivienda y Desarrollo Territorial 2010.

27. Revista Cenic Ciencias Químicas. (2005). Empleo de un producto Coagulante Natural para Clarificar Agua. Vol. 36. Especial. La Habana, Cuba

28. Romero Rojas, Jairo Alberto. (1998). Acuitratamiento por las lagunas de estabilización. Santa fe de Bogotá: Escuela colombiana de ingeniería.

29. Schwarz, D. (2000). Water clarification using Moringa oleiffera. Eschborn, Germany.
30. States, S., Tomko, R., Scheuring, M. \& Casson, L. (2002). Enhanced coagulation and removal of Crysptosporidium. J. AWWA. 94: 67- 77.

31. Stauber, J., Florence, L., Davies, C., Adams, M. \& Buchanan, S. (1999). Bioavailability of alin alum treatment drinking water. J. American Water Works Assoc. 91: 84-93.

32. Teixeira, P.C. (2003). Emprego da Flotação Por Ar Dissolvido no Tratamento de Efluentes de Lavagem de Veículos Visando a Reciclagem da Água. (tesis de Magister), Faculdade de Engenharia Civil, Universidade Estadual de Campinas, Campinas, Brasil.

\section{Conflicto de Intereses}

Los autores declaran no tener ningún conflicto de intereses 
UNAP 